\title{
Un análisis de la gestión de las competencias en el trabajo: el caso de los mandos intermedios de una factoría de carrocería y montaje de automóviles
}

\author{
Matilde MASSÓ \\ Departamento de Sociología \\ Universidad de A Coruña \\ m.masso@udc.es \\ Carlos LOZARES \\ Departamento de Sociología \\ Universidad Autónoma de Barcelona \\ carlos.lozares@uab.cat
}

Recibido: 5-10-2010

Aceptado: 25-01-2011

\begin{abstract}
RESUMEN
En este artículo se analizan aquellas orientaciones teóricas y prácticas de management basadas en la noción de competencia. En él se muestra que si bien la utilización de las competencias se sitúa claramente en la línea de la individualización de las relaciones laborales, en la práctica, la omisión de su carácter social y construido es susceptible de acentuar los efectos perversos de dicha individualización, minando en consecuencia la búsqueda de equidad salarial que supuestamente persiguen las empresas con la aplicación de este tipo de prácticas. En el estudio de caso que se presenta se analizan las diversas dificultades metodológicas relacionadas con el reconocimiento de las competencias en el trabajo, al tiempo que se formulan algunas propuestas definición que son susceptibles de matizar las consecuencias no queridas de la aplicación de estas prácticas.
\end{abstract}

Palabras clave: Competencias, individualización, relaciones laborales, relación de empleo, gestión de los recursos humanos.

\section{An analysis of management of competencies at work: the case of supervisors of a coachwork- assembly factory}

\begin{abstract}
This article analyse the theoretical perspectives and managerial practices focused on a competence based approach. We show that managerial uses of competencies increase the process of individualisation of industrial relations. At the same time, the omission of the social character of competencies may improve the non- intended consequences of the process of individualisation. It makes difficult to get the companies' goal of attaining a salary equity situation. We present a case study where we have analysed different methodological difficulties related to the process of identify and evaluate competencies. At the same time we propose some alternative definitions of competencies that may avoid the non- intended consequences of these kind of practices.
\end{abstract}


Keywords: Competencies, individualisation, industrial relations, employment relation, management, social actors.

\section{REFERENCIA NORMALIZADA}

Massó, M., Lozares, C. (2012). Un análisis de la gestión de las competencias en el trabajo: el caso de los mandos intermedios de una factoría de carrocería y montaje de automóviles. Cuadernos de Relaciones Laborales Vol. 30, núm. 1, p. 211-233.

SUMARIO: Introducción. 1. Las competencias en el trabajo: ¿La nueva ideología del management? 1.1 El "modelo de la competencia" desde el punto de vista de la gestión de los recursos humanos. 1.1.1. De la propuesta del management a la crítica sociológica. 2. Una propuesta teórico-metodológica para el estudio de las competencias. 2.1. La construcción social de las competencias. 3. Presentación del estudio de caso y metodología de la investigación. 3.1 La metodología: diseño, métodos y técnicas de investigación. 4. Análisis y resultados. 4.1. Breve contextualización del caso estudiado: algunos datos relevantes. 4.2. La profesionalización de los Jefes de Unidad. 5. Discusión de los resultados: La omisión de la dimensión social de las competencias. 5.1. La identificación de las competencias es el resultado de un "acuerdo" sobre el trabajo. 5.2. La competencia es un concepto dependiente de la situación y el contexto en que se desarrolla: Las condiciones de desempeño y evaluación. 6. Conclusiones. Bibliografía.

\section{Introducción}

Desde la década de los noventa la noción de competencia viene adquiriendo un notable protagonismo en el vocabulario de numerosas disciplinas tales como la sociología, las ciencias de la educación, la psicología o la administración de empresas. Si bien el origen del término se remonta a los primeros trabajos de la psicología diferencial de los años 60 (McClelland, 1973), su reapropiación como vocablo característico de los recursos humanos emerge en la década de los ochenta y se generaliza posteriormente, en la década de los noventa.

Precisamente, es el estudio de dicha reapropiación, expresada en el conjunto de las prácticas de management que toman la noción de competencia como objeto de gestión, lo que constituye el objetivo central de este artículo. Más específicamente, se trata de analizar la definición y los usos de la competencia que hace la empresa a partir de los métodos de evaluación de su plantilla.

El estudio de caso que se presenta se basa en una investigación ${ }^{1}$ realizada en una fábrica de carrocería y montaje de automóviles donde se ha aplicado una serie de prácticas basadas en la noción de competencia, orientadas a la gestión de los mercados internos del colectivo de los mandos de los talleres. En el artículo se

${ }^{1}$ Esta investigación ha formado parte de la tesis doctoral de Matilde Massó, presentada en la Universidad Autónoma de Barcelona en mayo del 2008 y dirigida por los doctores Carlos Lozares y Fausto Miguélez. La tesis se ha desarrollado con el apoyo del Centro QUIT y en el marco de los proyectos SEJ2005- 04078/SOCI y SEC2001-2543 del Ministerio de educación y ciencia y del Ministerio de Ciencia y Tecnología respectivamente. La tesis obtuvo el premio de Consell de Treball Económic i Social de Catalunya del año 2008. 
analizan las consecuencias no deseadas derivadas de no tomar en consideración el carácter social y construido de la competencia. En este sentido, se pone de relieve el papel jugado por cada uno de los actores sociales "afectados" por este tipo de prácticas, así como también las transformaciones de la relación de empleo que conlleva la utilización de las competencias como elemento organizador de las políticas de recursos humanos.

\section{Las competencias en el trabajo: ¿la nueva ideología del management?}

Los actuales usos empresariales de la competencia están asociados a un conjunto de prácticas en materia de organización del trabajo y gestión de los recursos humanos basadas en una evaluación individualizada de los trabajadores. Dichas prácticas introducen nuevas reglas en el significado de la relación salarial que tienen que ver con la clasificación de los trabajadores y los salarios. Ahora bien, ¿cuál es la especificidad de estas prácticas según los teóricos del management? ¿Cómo influyen dichos aspectos en los nuevos significados otorgados a la prestación laboral?

\subsection{El "modelo de la competencia" desde el punto de vista del management}

El denominado "modelo de la competencia" ha de entenderse en el marco de la creciente importancia otorgada a los recursos humanos como vector de crecimiento y fuente de ganancias de productividad. Desde la década de los 80 asistimos a un cambio en las estrategias organizativas y manageriales que, entre otros factores, ha comportado el desarrollo y proliferación de nuevas prácticas y teorías basadas en la reorientación de los recursos internos de las empresas. La atención sobre "viejos" pero a la vez "nuevos" aspectos como son la motivación, la implicación, la participación, la mejora del desempeño, se generalizan en esta década tanto en EEUU como en Europa (Hammer \& Champy, 1997; Kanter, 1999; Peters, 1993). Dicha expansión se produce en un contexto marcado por la acentuada competencia internacional y la saturación y contracción de los mercados occidentales de bienes y servicios. Todo ello empuja a las empresas a buscar ventajas competitivas basadas en nuevas prácticas de carácter menos jerárquico y más orientado a la búsqueda de motivación e implicación de los trabajadores en una "empresa común" o una "empresa liberada" (véase, entre otros, el análisis crítico realizado por Willmott, 2007). Siguiendo a Fernández Rodríguez (2007), la incertidumbre de los mercados se traslada a los propios trabajadores, responsables de garantizar su propia empleabilidad, esto es, la permanencia en un empleo a través de la formación continua y la adherencia a los valores corporativos.

Es evidente que la atención prestada a los recursos humanos en tanto que fuente de ventaja competitiva no constituye un hecho totalmente novedoso. Con todo, la diferencia entre la gestión por competencias y otras prácticas características de los ochenta es la formalización y aplicación de una serie de procesos basados en conceptos como la implicación personal, la adhesión de la subjetividad y las motiva- 
ciones intrínsecas de los trabajadores (Alonso, 2007; Brunet, 2007; Fernández Rodríguez, 2007; Willmott, 2007).

Estos conceptos se derivan, en gran medida, de estudios teóricos y empíricos que resaltan el papel de los recursos humanos en la generación de una ventaja competitiva sostenida (Barney, 1991) y se enmarcan en lo que se conoce como la "Teoría de Recursos y Capacidades". Según ésta, los trabajadores o más precisamente sus competencias dejan de ser un mero coste que han de soportar las empresas y se convierten en un aspecto central de sus recursos en la medida que son susceptibles de proporcionar una ventaja estratégica (Grant, 1995; Hamel y Prahalad, 1994).

Más recientemente, otros enfoques del management han hecho hincapié en la propuesta de modelos específicos para la gestión de los activos intangibles (fundamentalmente competencias tácitas y conocimientos) que generan valor añadido a la empresa (Nonaka et al. 2000). La mayoría de estos intangibles tienen que ver con la captación, estructuración y transmisión del conocimiento procedente tanto del interior como del exterior de la empresa, posibilitando a la misma alcanzar una posición competitiva en el mercado. Como afirma críticamente Alonso (2007), el conocimiento pasa a convertirse en una mercancía, y una herramienta fundamental de desregulación social en el discurso del management postmoderno, al disolver el conocimiento colectivo en competencias individuales. De esta manera, el conocimiento pasa a ser el recurso de mayor importancia de las empresas en las luchas del tipo "todos contra todos".

Veamos más concretamente cómo se formalizan estas propuestas del management y sus implicaciones en la evolución de la relación de empleo.

\subsubsection{De la propuesta del management a la crítica sociológica}

Los usos y concepciones de la competencia no son uniformes en la literatura del management, sino que varían en función de las perspectivas, autores e incluso empresas que los formulan.

Una primera concepción de la gestión por competencias está presente en aquellas perspectivas que inciden en los aspectos emocionales y simbólicos que resaltan la condición de miembro de una organización, y por tanto, la pertenencia a un grupo humano con una identidad diferenciada (Hamel y Prahalal, 1994). La interiorización de las formas de control y la búsqueda de un compromiso basado en la voluntad de realizar de manera excelente el propio trabajo se encuentran en el centro de este tipo concepciones y prácticas. La novedad significativa reside, primero, en tratar de identificar y premiar las conductas esperadas, antes que las tareas prescritas, relacionadas con un rendimiento "óptimo" o "superior" de los trabajadores y segundo, en vincular dichas conductas con los valores corporativos de la empresa. Precisamente, la CEOE (2002) considera que uno de los aspectos más relevantes del modelo de la competencia es la capacidad de adecuar y alinear a los trabajadores con los objetivos estratégicos de la empresa.

Esta búsqueda de identificación con los valores corporativos comparte, según Reynaud (2001), el sueño de una antigua utopía capitalista: la conversión del trabajador en un subempresario de su propia mano de obra. En efecto, el trabajador ya 
no sólo ha de poner al servicio de la empresa su saber y experiencia a cambio de un salario, sino que, en lo sucesivo, se verá obligado a aportar un resultado de una determinada manera, es decir, poniendo en práctica sus conocimientos, aptitudes y actitudes a cambio de una empleabilidad. Este término hace alusión precisamente al conjunto de competencias que brindan a un individuo la oportunidad de ingresar en un empleo y permanecer en él (Boltanski y Chiapello, 2002). Dicha empleabilidad aumenta en la medida que el trabajador adquiere nuevas competencias o desarrolla las que ya posee poniéndolas al servicio de la empresa.

En segundo lugar, la utilización de la noción de competencia como herramienta de management se concibe como un indicador del reconocimiento del trabajo real o efectivo. En este sentido, diversos autores (Boam y Sparrow, 1992; Blanco Prieto 2007; Lichtenberger 2003; Zarifian, 1999) consideran que la noción de competencia favorece la posibilidad de reconocer "la inteligencia productiva" que los trabajadores ponen al servicio de la empresa en el desempeño diario de su trabajo. Al mismo tiempo, los teóricos del management afirman que las prácticas basadas en la noción de competencia garantizan el reconocimiento de las aportaciones individuales de cada trabajador y una valoración más objetiva de las personas que aportan sus conocimientos a la empresa (Jiménez, 2000; Tremblay y Chenevret, 1998).

Sin embargo, estas prácticas no conducen a un mayor reconocimiento del "trabajo real". La selección y evaluación de competencias clave constituye un proceso complejo como es el de la traducción al registro de la actividad, de las capacidades requeridas para desarrollar dicha actividad. Un proceso que puede generar y genera la ilusión de objetividad utilizando simplemente y sin un juicio crítico y pragmático, las técnicas de selección y evaluación elaboradas por expertos. De hecho, como recuerda Grugulis et al. (2004), la importancia otorgada a determinadas competencias no implica necesariamente que el contenido del trabajo haya cambiado, ni tan siquiera que las nuevas competencias demandadas vayan a ser remuneradas o reconocidas en los sistemas clasificación. En este sentido, la apelación del management a la movilización de valores personales representa una evolución de las formas de control empresarial pero no un enriquecimiento del trabajo, ni tan siquiera un nuevo reconocimiento del trabajador.

Finalmente cabe señalar aquellas propuestas centradas en subrayar lo que comporta dicho modelo de gestión y que implicarían un supuesto cambio de paradigma en el campo de la organización y gestión de los recursos humanos. Dicha transformación es interpretada, en numerosas ocasiones, en términos de ruptura con respecto a los modelos organizativos y gestionarios vigentes y se concretaría en el paso de un modelo basado en el puesto de trabajo -job-based organization- a otro basado en las competencias -competency- based organization- (Bernard et al., 1998; Blanco Prieto, 2007; Giorgini, 2000; Lawler, 1994; Zarifian, 1999). En el primer modelo, los puestos de trabajo son las unidades básicas del diseño de las distintas políticas de recursos humanos según una particular división del trabajo. En el segundo, 
de carácter más flexible, dichas unidades son las competencias o agregados de competencias ${ }^{2}$. Para Lawler (1994), el modelo organizativo y gestionario dominante durante décadas ha sido el basado en el paradigma del puesto de trabajo que se presentaba en coherencia con el objetivo de incrementar la racionalización de los tiempos y los recursos disponibles para así obtener la máxima productividad y eficiencia. Dicho modelo se considera en la actualidad, como excesivamente rígido y estático e incapaz de proporcionar respuestas satisfactorias ante los nuevos retos del contexto socioeconómico. Para Lawler (Ibíd.), la alternativa al paradigma organizativo del puesto de trabajo susceptible de proporcionar una ventaja competitiva, es el diseño de sistemas organizativos en los que las competencias de los individuos constituyen el elemento básico que facilita el desarrollo organizacional.

Oiry y D'Iribarne (2001) analizan críticamente este tipo de propuestas, dado que los usos empresariales de la competencia se conciben como el producto de una racionalidad única exterior a las empresas que impone determinadas respuestas que son interpretadas en clave de necesidad. Ambos autores hablan de la llamada "cadena lógica" del discurso empresarial según la cual las desregulaciones en materia de organización y gestión de los recursos humanos se legitiman y justifican por las transformaciones inmanentes del contexto socioeconómico.

\section{Una propuesta teórico-metodológica para el estudio de las competencias}

Frente a las ventajas que la utilización de las competencias aporta al management, se han de poner también de relieve los posibles sesgos y consecuencias no deseadas derivadas de no considerar el carácter social, contextual y situado de las competencias; sesgos y consecuencias frecuentemente olvidadas en las prácticas de gestión de los recursos humanos. Como veremos, la conciliación de la recompensa al mérito individual asociada a la gestión por competencias y la búsqueda de un funcionamiento colectivo óptimo es un proceso altamente complejo. La utilización de la competencia introduce una lógica de diferenciación susceptible de debilitar los vínculos de solidaridad existentes en el seno de un equipo de trabajo, precisamente por privilegiar la eficacia del reconocimiento individual, en detrimento de un reconocimiento igualitario e impersonal de todos los miembros de un equipo de trabajo (Massó, 2007, 2009). La definición de la competencia que se propone en este apartado hace hincapié precisamente, en el carácter contextual y situado del término como mecanismo para superar dichas limitaciones.

Los fundamentos teóricos que sustentan esta propuesta, como en buena parte se viene señalando, se basan en una pluralidad de investigaciones desarrolladas en el campo de la sociología del trabajo y de las organizaciones. Siguiendo la perspectiva

\footnotetext{
${ }^{2}$ Para un análisis crítico de los fundamentos empíricos y teóricos de dicha contraposición, véase Massó $(2007,2009)$.
} 
abierta por Pierre Naville (véase Tanguy, 1986) y varios de sus seguidores (Alaluf, 2003; Rolle, 2004; Stroobants, 2005) nuestro presupuesto teórico se basa en abordar el trabajo como una relación social en situación y contexto. En consecuencia, la interacción y por tanto la relación ha de ser la unidad mínima de análisis no sólo en la investigación sino también en todos los proyectos de evaluación de los procesos de trabajo. Ello incluye los contextos, los resultados de las relaciones, negociaciones y conflictos entre los distintos actores implicados, que precisamente también condicionan dichos procesos (García et al., 2005; Lozares, 2007). En este sentido, nociones centrales como la cualificación o la competencia, no se han de reducir a atributos individuales y técnicos, cuyo análisis se circunscriba de manera estable a la situación de trabajo, sino que ambas nociones se han de comprender como proceso y resultado de una valoración y estratificación social, al ser herramientas vigentes que clasifican a los trabajadores y los salarios. Ello es un aspecto señalado ya por diversos autores españoles que definen la cualificación como una construcción social, vinculada a una determinada división del trabajo y al contexto sociolaboral específico de cada Estado (Castillo et al., 2000; Homs \& Prieto, 1991; Miguélez, 1993)

A su vez, este artículo se basa en las diversas aportaciones realizadas por autores que plantean un análisis crítico de la literatura managerial, tales como Boltansky \& Chiapello (2002), o las diversas aportaciones realizadas desde los Critical Management Studies (Fernández Rodríguez, 2007), todos ellos centrados, entre otros aspectos, en el análisis de las relaciones entre el poder y las prácticas discursivas en la empresa moderna (Gómez Rodríguez \& Lopez Aranguren, 2004).

\subsection{La pertinencia social de las competencias}

En este artículo se entiende la noción de competencia como una combinación de conocimientos ("saber"), aptitudes y experiencia ("saber-hacer"), y comportamientos ("saber- estar"), que se activan, aplican y por tanto se manifiestan, en el contexto y situación precisos de su ejercicio. La competencia no se aprecia en sí misma si no a través de la acción y por tanto, su visibilidad es inseparable de las condiciones en las que es aplicada, medida, y especialmente evaluada (Lichtenberger, 2003).

Dado que la competencia es reconocida y validada en su puesta en práctica, en una situación y contexto de trabajo, su naturaleza no es simple, sino que está articulada o relacionada con los distintos niveles contextuales que la configuran, las prácticas interactivas y los artefactos que intervienen en dichas interacciones. A su vez, en los procesos de evaluación de las competencias también se manifiesta esa dimensión relacional ya que es cuando las competencias se visibilizan a través de su medición y valoración. En la figura 1 se expone esquemáticamente el contenido articulado de la noción de competencia tal y como se concibe en este artículo: 
Figura 1. Definición de Competencia

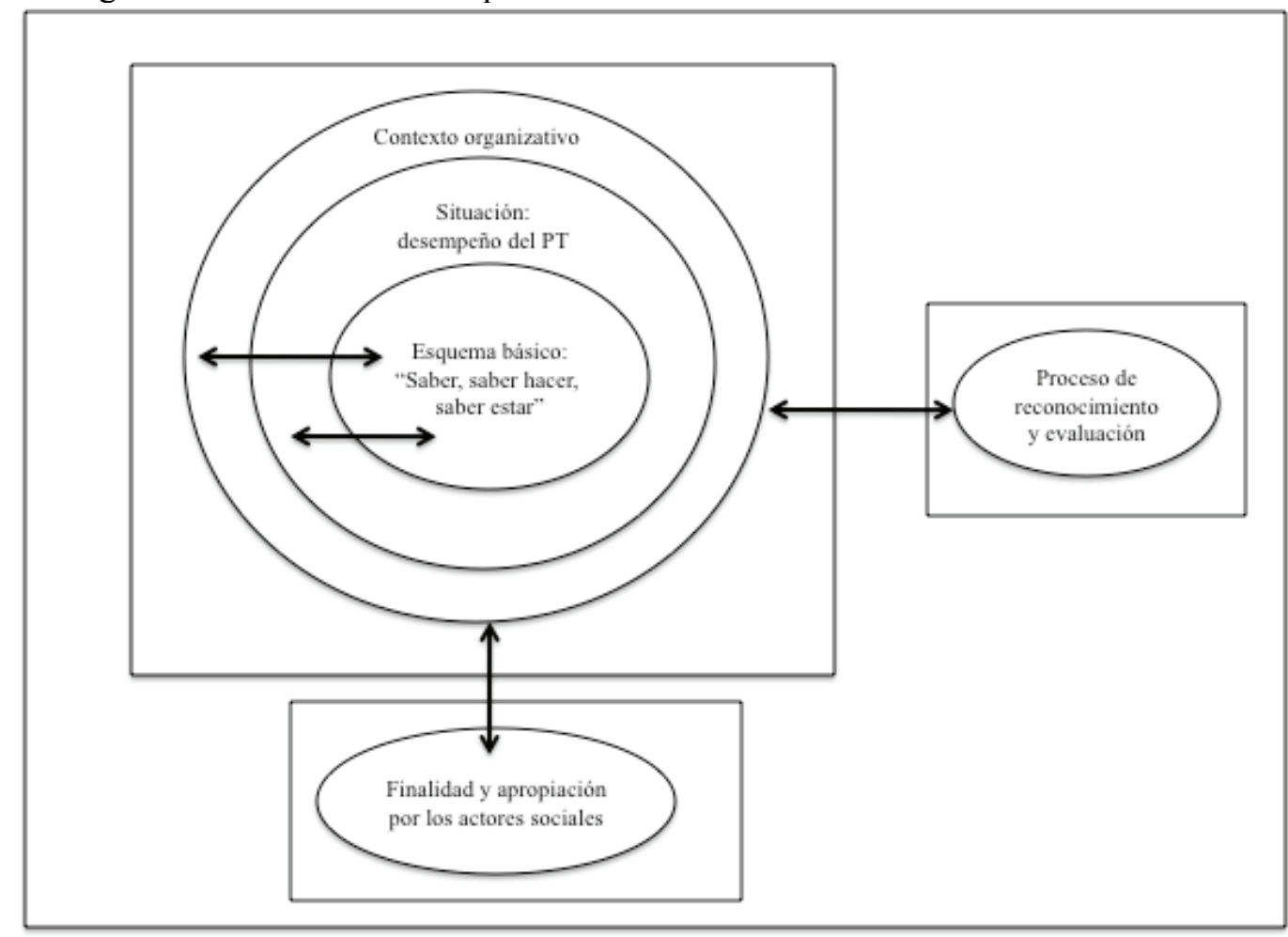

Fuente: elaboración propia

El núcleo de la competencia está constituido por la combinación y articulación de los conocimientos, las aptitudes y los comportamientos o actitudes. Dichos aspectos se han denominado en la figura 1 "Esquema básico de la competencia". Cabe señalar que estos componentes no constituyen por sí mismos competencias, sino que son aspectos o parte de la misma (Levi-Leboyer, 2002; Stoof et al., 2002; Van Loo \& Semeijn, 2004), cuyo núcleo consiste, precisamente, en la capacidad de movilizar y poner en práctica estos recursos.

Por otro lado, este "núcleo duro" de las competencias se vincula a una determinada tarea o situación espacio- temporal e interactiva de trabajo, en el contexto específico de una organización caracterizada por unas reglas de funcionamiento y una cultura específica. Es decir, se articulan en lo que en el esquema de la figura 1 se han denominado "Situación" y "Contexto", subrayando su carácter situado en la práctica cotidiana del trabajo, en el seno de un equipo y una organización más amplia.

Tal y como se ha apuntado anteriormente, la identificación y evaluación de las competencias exige tener en cuenta una serie de condiciones. En primer lugar, la distinción entre rasgos de personalidad y competencias es a veces una frontera un 
tanto difusa. Tal y como recuerda Bellier (2002), la competencia se refiere a la capacidad para alcanzar determinados objetivos o solucionar determinados problemas asociados a las situaciones cotidianas de trabajo y no a lo que las personas "son", esto es, a sus rasgos de personalidad. Con todo, ambos campos están estrechamente relacionados, lo que hace que la validación de las competencias sea un proceso susceptible de incluir sesgos y arbitrariedades que influyan en su resultado.

En este sentido, es pertinente preguntarse si la unidad de análisis o el sujeto de las competencias, es el individuo, la situación, el grupo de trabajo, o todo ello. La cuestión es importante porque una $u$ otra opción exige metodologías diferentes de análisis y en consecuencia, métodos de evaluación diferentes por parte de la empresa; y porque el papel otorgado al contexto y concretamente, al equipo de trabajo y a la organización, será diferente según consideremos una u otra opción.

En nuestro modelo se opta por una concepción interactiva y distribuida de la noción de competencia. Esta perspectiva considera que los distintos actores no pueden contemplarse aislados sino interdependientes. En este sentido, la competencia es considerada como un espacio conceptual en el que se articulan dos lógicas: la del individuo y la de la organización y el contexto, lo que significa, en consecuencia, que una evaluación equitativa no puede ser más que una evaluación simultánea del individuo, de la organización y de la situación en la que desarrolla su trabajo (Combes 2004).

En segundo lugar, la identificación de las competencias no constituye un proceso mecánico, sino que es el resultado de un acuerdo sobre el trabajo. Una de las etapas fundamentales de este tipo de prácticas es la de selección e identificación de las competencias consideradas claves y de los criterios que permitirán su validación. En esta línea entendemos que la competencia puede tener contenidos diferenciales cuyo sentido depende de las relaciones de fuerza de los actores implicados, o bien de la existencia de acuerdos entre ellos. Ello implica que no todas las competencias que son necesarias para realizar una tarea son relevantes para la empresa que, en última instancia, es el actor más fuerte. Este aspecto se ha denominado en la figura 1 "Finalidad y apropiación de las competencias por los actores sociales". Es evidente que si uno de los actores se apropia e impone una interpretación, el riesgo de provocar efectos perversos sobre el resto de los actores aumenta. De alguna manera hay un doble juego de validez conceptual, la búsqueda de una objetivación de base y un equilibrio consensuado de significados entre los distintos actores sociales (Stoof et al, 2002)

Como se observa, el problema fundamental que plantea la definición de competencia no radica en el análisis de una semántica de base sobre su identidad, sino en la inclusión de otros ingredientes sustantivos que contribuyen también a darle sentido y que tienen que ver con su carácter relacional y con los procesos y técnicas de medición. 


\section{Presentación del estudio de caso y metodología de la investigación}

El caso que presentamos en este artículo se centra en una factoría de carrocería y montaje de automóviles. Concretamente se ha analizado el colectivo de los mandos intermedios de dos talleres (Montaje y Soldadura) que tienen a su cargo un grupo de unas 15 o 20 personas. Este colectivo se ha visto afectado por la introducción, a iniciativa de la dirección central de recursos humanos y sin contar con un proceso de negociación con los sindicatos, de una serie de prácticas basadas en la noción de competencia que se han englobado bajo el nombre de "Profesionalización de los Jefes de Unidad". Este proceso ha constituido un paso estratégico de la empresa para introducir progresivamente un cambio de mayor envergadura, basado en un modelo de la competencia que afectará al resto de colectivos de la factoría (mandos superiores y grupo obrero), implicando la modificación del sistema de clasificación profesional y, en consecuencia, de la determinación del salario y la organización del sistema de promoción profesional.

Teniendo en cuenta este marco, el objetivo de la investigación consistió en identificar y analizar la definición de la competencia propuesta por la empresa como también los efectos sociales que ha comportado su utilización como instrumento de gestión. Más concretamente, se examinan los costes sociales que han implicado este tipo de prácticas, teniendo en cuenta el significado que los distintos actores sociales atribuyen a la identificación y evaluación del concepto de competencia y que explican en gran parte los distintos modos y estrategias de acción tanto empresarial como sindical.

\subsection{La metodología: diseño, métodos y técnicas de investigación}

La estrategia metodológica seguida se enmarca en un diseño de tipo explicativointerpretativo, cuyo principal objetivo es proponer un marco genérico y al mismo tiempo operativo sobre los conceptos y modelos tratados, referidos a la competencia, su funcionamiento, y al mismo tiempo, identificar el significado que les atribuyen los distintos actores sociales. La orientación metodológica adoptada se basa, por un lado, en el estudio comparativo de casos (Yin, 1994; Coller, 2005; Ragin \& Becker, 1992; Stake, 1994) y por otro, en lo que diversos autores han denominado una aproximación multiestratégica (Lozares et al., 1998; Valles, 1997).

Los objetivos de esta investigación, de la que aquí se expone una parte, han exigido una aproximación metodológica de carácter cualitativo y micro-social que posibilitase un conocimiento directo de la organización y funcionamiento de la empresa seleccionada, así como también de los procesos de trabajo desarrollados en los talleres. Para conjugar el grado de complejidad de cada uno de estos factores, la estrategia del estudio de casos nos ha permitido articular los diferentes planos u enfoques, así como integrar la naturaleza diversa de las distintas fuentes de información utilizadas. Precisamente, ello es lo que se ha denominado una metodología multiestratégica (Lozares et al., 1998), que constituye un principio básico para reforzar la fiabilidad y la validez interna de la investigación durante el trabajo de campo, dado el recurso que se ha hecho de la utilización de diversas técnicas para 
analizar y medir el mismo fenómeno. Para el caso que aquí se presenta, las distintas fuentes utilizadas han sido las siguientes:

En primer lugar, un tipo de información procedente de la observación directa no participante de los procesos de trabajo (las Unidades Elementales de "Grupo Motor Propulsor" y "Lado de Caja Derecho") y de los entornos y contextos más inmediatos a dicho procesos. En este caso se han realizado 8 sesiones de observación directa de carácter semiestructurado, en el marco de un contrato laboral en la factoría de una duración de 2 meses y medio en el área de recursos humanos. El tipo de registro se ha realizado mediante la toma simultánea de notas y en numerosos casos, posterior al curso de la observación.

Como se ha mencionado anteriormente, a pesar de la alta riqueza informativa de las observaciones nunca es suficiente incluso la exhaustividad del análisis e interpretación para alcanzar plenamente los objetivos de la investigación. Otra de las técnicas utilizadas fue la entrevista a los distintos actores implicados: los JU, sindicatos y representantes de la dirección de recursos humanos, cuadros medios y superiores, etc. En total se realizaron un total de 27 entrevistas semiestructuradas registradas en su mayoría con grabadora. A su vez, también fue clave aquella información procedente del material documental elaborado por la dirección de la empresa o las principales organizaciones sindicales (convenios colectivos, acuerdos, actas de reuniones, organigramas, artículos, datos o informes de Intranet, etc.)

La combinación de todas estas fuentes de información nos ha permitido el desarrollo de líneas de investigación convergentes (Yin, 1994) y el aumento del grado de validez cualitativo del diseño de la investigación.

\section{Análisis y resultados}

\subsection{Breve contextualización del caso estudiado: algunos datos relevantes}

La factoría estudiada pertenece a una empresa (denominada "X" para mantener el anonimato), que a su vez forma parte de un grupo automovilístico multimarca. Éste posee alrededor de 33 fábricas de carrocería- montaje y de motores situadas mayoritariamente en Europa y Asia. Según datos del 2006, la plantilla del grupo es de un total de 126.584 trabajadores, teniendo en cuenta los centros administrativos (excluyendo la red comercial) y las distintas plantas de producción. La factoría estudiada cuenta con una plantilla de 2818 personas de una media de edad de 48 años, según datos del año 2006. El 82,5\% de la plantilla se concentra en el área de fabricación y, concretamente, el $40,16 \%$ del total de la plantilla forma parte del departamento de Montaje, que en comparación con otros talleres de la fábrica, posee un bajo grado de automatización.

La planta de producción está especializada en la fabricación de vehículos de gama media. En la actualidad de sus líneas de fabricación salen tres versiones del mismo coche, dos de ellos en exclusiva mundial. Todo ello hace que la variabilidad del producto sea considerable y, en consecuencia, también lo sean los modos operativos que ha de dominar el obrero. Además, la fabricación en serie se adapta a las 
exigencias del comprador, es decir, los vehículos se montan en función de las características técnicas y de diseño elegidas por el cliente.

En cuanto al proceso de producción, la factoría estudiada abarca todas las fases del proceso de fabricación del automóvil, que se organiza en 5 talleres (Embutición, Soldadura, Pintura, Montaje y Logística), en los que se realiza una parte del proceso de elaboración y montaje de la carrocería. La planta cuenta con instalaciones altamente modernizadas, que permiten una producción media de un vehículo por minuto y una cadencia de 1100 vehículos por día. El modelo productivo de la factoría se enmarca en lo que diversos autores (Olivier et al., 2001; Womack et al., 1994) denominan el paradigma de la "Producción Ligera" (Lean Production), caracterizado entre otros aspectos, por la aplicación del sistema JAT relativo al suministro de piezas y módulos. El JAT parte de un modo de fabricación adaptado al cliente, denominado usualmente Built to order en el que el objetivo esencial es que la producción fluya a un ritmo continuo (flujo tenso) que no está marcado por la capacidad productiva de las máquinas sino por las demandas de los clientes. Este principio rige no sólo entre las escalas de los proveedores sino también entre los equipos de trabajo y las "unidades de negocio" dentro de las fábricas.

En cuanto a la organización del trabajo, en la década de los 90 la empresa X. inicia un importante proceso de "modernización" y reorganización productiva. En la planta estudiada este proceso se ha concretado en la organización por Unidades Elementales de Trabajo (UET) (véase, Castillo, 1998, 2000) que constituyen equipos de tamaño reducido bajo una responsabilidad jerárquica única, un mando intermedio denominado "Jefe de Unidad" (JU).

La organización en UET ha sido paralela a la reducción de niveles jerárquicos (el paso de 7 a 4 categorías de mando) y la redefinición de los roles del conjunto de la cadena de mando. Las distintas personas entrevistadas han coincidido en apuntar que el JU (generalmente los antiguos "Encargados de Sección") es la figura que más ha ampliado sus funciones con la creación de las UET. El JU debe planificar y gestionar su actividad y la de los trabajadores que tiene a su cargo para alcanzar los resultados y objetivos propuestos en materia de calidad, costes, recursos humanos, estandarización y plazos. En este sentido, el JU posee una elevada autonomía en la gestión de su propio equipo de trabajo y de los medios de producción que la UET necesita diariamente.

Este proceso de reorganización productiva coincide con una profunda renovación en las políticas de gestión de los recursos humanos con el objetivo de "ajustar" la plantilla cualitativa y cuantitativamente. Un ejemplo de este proceso es la progresiva profesionalización de la plantilla, que por su importancia analizaremos con detalle en los siguientes apartados.

\subsection{La profesionalización de los jefes de unidad}

La profesionalización de los JU constituye una iniciativa que emerge de la dirección central de recursos humanos a nivel del grupo y se aplica de manera unidireccional, aunque con ritmos diferentes, a todos los centros de trabajo. La base de este proceso ha sido la construcción de un listado de competencias, concebido como una 
herramienta clave para gestionar las condiciones de acceso a las funciones de JU y JU de Primera, esto es, definir un modelo de carrera profesional para los mandos intermedios, una nueva política de ascensos basada en la evaluación de las competencias por parte del mando jerárquico correspondiente y delimitar con claridad las competencias específicas que ha de desempeñar este colectivo.

Se ha de señalar que dichas prácticas se enmarcan en un proceso más amplio que afecta al resto de los colectivos de fabricación, mantenimiento y logística. Con ello, la empresa busca unificar los niveles de productividad de las distintas fábricas del grupo, mediante la creación de un referencial o listado de competencias para los tres primeros niveles clasificatorios (los obreros, los JU y los Jefes de Taller). En el caso de los centros españoles se comienza a poner en práctica la profesionalización de los JU (y no del grupo obrero, por ejemplo) debido a los cambios que comportaba, para este colectivo, la nueva organización en UETs. A su vez, se ha de resaltar el mayor margen de maniobra que posee la empresa a la hora introducir modificaciones en las políticas que afectan a los mandos intermedios en comparación con el resto de categorías más bajas, para las que existe un sistema de carácter más regulado y controlado sindicalmente.

En el caso concreto de la fábrica estudiada, estas prácticas tienen como objetivo manifiesto lograr una "adecuación más eficiente de los recursos humanos a las necesidades presentes y futuras de los talleres ${ }^{3 "}$ e iniciar una nueva etapa en las políticas de gestión de los recursos humanos basada en dos ejes estratégicos: la individualización de la gestión de los recursos humanos y la descentralización del management. Veamos cada uno de estos aspectos con más detalle.

En primer lugar, la profesionalización de los JU ha implicado la elaboración de un listado de competencias, que pasa a constituir la herramienta clave de definición, valoración del trabajo y clasificación de este colectivo, en función de las puntuaciones obtenidas en la evaluación de las competencias. La profesionalización de los JU ha de entenderse como un primer paso para un cambio de mayor envergadura basado en la creación de un nuevo sistema de clasificación profesional que se asienta en el reconocimiento de una serie de "bloques de competencias" adquiridas y realmente ejercidas. El nuevo sistema comporta la pérdida de garantías colectivas asociadas al antiguo concepto de cualificación. Dichas garantías se manifestaban, para el caso concreto del encuadramiento profesional, a través de la negociación colectiva de una serie de criterios objetivables (por ejemplo, la antigüedad o la formación), externos a la situación de trabajo, y en el que los sindicatos ejercían un papel de control, altamente regulado mediante el convenio colectivo.

En segundo lugar, este proceso comporta un cambio sustancial en el modelo de gestión de la promoción profesional. En el caso de la promoción de Oficial de primera a JU, las nuevas prácticas basadas en la noción de competencia sistematizan una serie de procesos de promoción/ selección que hasta la fecha se limitaba a

\footnotetext{
${ }^{3}$ Jefe de Recursos Humanos de la factoría
} 
una prueba de concurso examen para pasar de "Oficial de Primera" a "Encargado de Sección" (los actuales JU). En el caso de la promoción de JU a JU de Primera, las nuevas prácticas inauguran la posibilidad de acceder a una nueva categoría laboral, la de JU de Primera (que no comporta un aumento de responsabilidad) en función de la puntuación obtenida en la evaluación de las competencias.

En ambos casos, el cambio operado apunta a una descentralización de las decisiones del management, en el sentido de que son los mandos directos los encargados de identificar, formar y evaluar a los potenciales JU y JU de Primera. A su vez, estas prácticas suponen, con respecto a la gestión de los puestos de trabajo, la introducción de una regla de promoción cuyo contenido se configura mediante un juicio local elaborado por el mando superior jerárquico correspondiente.

Finalmente, el proyecto de profesionalización supone la aplicación de un sistema de remuneración variable dependiente de la valoración de las competencias ejercidas y adquiridas. La prima por desempeño, denominada popularmente "la Bufanda", se reconoce en el convenio colectivo a través del concepto variable denominado "prima personal". En la actualidad, el importe total de la prima (que varía entre 0 y 2300 euros al año) viene determinado por la evaluación del referencial de competencias y la entrevista anual individual, donde precisamente se comentan los resultados de la evaluación de competencias y la consecución de los objetivos asociados a la UET.

\subsection{El referencial de competencias elaborado por la empresa. Características relevantes}

El referencial o listado de competencias de los JU se organiza en siete familias de competencias, treinta y cuatro competencias requeridas, sesenta y dos criterios de validación y setenta y ocho indicadores. Semejante referencial indica que la operativización de las distintas competencias se ha realizado con un alto grado de detalle, con el fin de capitalizar la experiencia de los mandos intermedios y transferir los métodos y "el saber hacer" mediante los estándares compartidos por todas las factorías del grupo.

Teniendo cuenta que no ha sido posible presentar el referencial completo por considerarse una herramienta confidencial para la empresa, se ha de señalar que las competencias requeridas constituyen un registro exhaustivo de las actividades más relevantes que han de realizar los JU para cumplir con los objetivos de Calidad, Coste, Plazos, Estandarización y Recursos Humanos. Al respecto, llama la atención el monopolio de la empresa en la identificación de dichas competencias que, como se ha comentado, ha sido una propuesta centralizada de la dirección del grupo. Este hecho explica el tipo de referencial elaborado, cuyo objetivo es más bien prescribir las actividades y los métodos empleados por los JU, sea cual sea el taller y la factoría al que pertenezcan, y aún cuando su trabajo se caracterice por la reacción ante los imprevistos, antes que por la prescripción de las tareas.

Como puede deducirse de la definición de competencias que proponemos en este artículo, las nociones de actividad y competencia son de naturaleza diferente. Por tanto, aunque la competencia se manifieste a través de la acción no son equivalentes; 
la competencia es la una capacidad cognitiva y fáctica que permite a los individuos comprender, anticipar y resolver los diversos problemas y situaciones en el proceso del trabajo. En este sentido, aunque las competencias se expresan, aplican o realizan directamente en la práctica y situación de trabajo, son susceptibles de identificarse y clasificarse en un esquema conceptual y metodológico que permite describirlas y diferenciarlas. La inducción en el modo de conceptualizarlas facilita a su vez operativizarlas y vincularlas a un resultado y nivel de desempeño del trabajo.

En la UET de Montaje analizada, la gestión de los recursos humanos ocupa un lugar central, dado que, en comparación con otros talleres, el nivel de automatización no es significativamente elevado y se requieren más operarios por línea, al tiempo que el coeficiente de absentismo es significativamente elevado llegando a un $6,5 \%$ a fecha de mayo del 2006. El JU ha de dominar numerosas herramientas de Management que se utilizan en todas las factorías del grupo, como por ejemplo, el portal de la Base Unificada de Personal, que se actualiza al inicio de cada jornada y se utiliza para el control de la plantilla; el "cuadro ILU", donde se gestiona la formación y la polivalencia de los operarios; o el "Cuadro de Exigencias Operatorias", donde se definen las distintas operaciones de los puestos de trabajo y los conocimientos y competencias necesarias para desarrollarlos. Al mismo tiempo existen numerosas herramientas para desempeñar la función de "animar", motivar y realizar el seguimiento de los miembros de la UET como las entrevistas individuales o las reuniones de equipo.

Si bien estos aspectos específicos y técnicos están reconocidos en el referencial de competencias, éste omite la importancia de todos aquellos recursos relacionales que tienen que ver con la capacidad de comunicación y liderazgo, así como con la capacidad resolutiva y analítica de todos los problemas relacionados con la gestión de la mano de obra. El más importante de estos problemas es la gestión del absentismo, no sólo de su propia UET, sino de otras denominadas "cliente o proveedor" con las que el Jefe de Unidad tiene un estrecho contacto:

Normalmente lo que tratas es de planificar la situación del día siguiente. Lo que haces es gestionar tus bajas con los otros Jefes de Unidad de tu taller. Recuerdo que la vez más grave que tuve fue una mañana en que estaba sustituyendo a un JU del taller y faltaron en su línea 6 personas. Fue horrible, casi se para la cadena ${ }^{4}$

Por otro lado, la gestión de los recursos humanos exige, como se ha señalado, una alta capacidad de liderazgo basado no en la mera obediencia, sino en la búsqueda de implicación y motivación del equipo:

Aquí no tenemos la potestad de coger a la gente que nosotros queremos, y ello agrava la situación (...) Tú tienes 30 puestos, 30 operarios. Cada uno con un carácter y una edad diferente. A estas alturas ya les conozco bien y cada uno ya está más o

\footnotetext{
${ }^{4} \mathrm{JU}$ de Montaje.
} 
menos bastante bien adaptado a cómo desempeña su labor. Ahora estamos en una


los hilos, o por suerte. Yo lo hago intuitivamente ${ }^{5}$.

El aspecto "intuitivo" al que se refiere el JU, tiene que ver claramente con un estilo de management personalizado y el despliegue de una serie de mecanismos nada "intuitivos" que le permiten gestionar y anticiparse a las incidencias cotidianas, como por ejemplo, los planes de formación continuada, la gestión de la polivalencia y la rotación por distintos puestos de trabajo de los miembros de la UET. En el caso del taller de Soldadura, sin embargo, la gestión del absentismo no es tan importante (se trata de un taller altamente automatizado), mientras que sí lo es el intento de motivar e implicar a las personas que llegan cedidas de la fábrica de motores, debido a la disminución de la producción y de las ventas del coches. En ambos casos, el papel del JU es clave a la hora de crear un buen clima social de cooperación, consiguiendo resolver los problemas producción, mantenimiento, calidad, costes, etc. en el mismo terreno de trabajo, sin trasladárselos al Jefe de Taller.

\section{Discusión de los resultados: la omisión de la dimensión social de las compe- tencias}

La profesionalización de los JU y sus efectos en la relación de empleo, ha provocado numerosas consecuencias no deseadas en las relaciones sociales de trabajo. Ello se ha debido a no haber tenido en cuenta los elementos contextuales que interfieren en la evaluación de las competencias y las expectativas e intereses que los distintos actores sociales poseen con la aplicación de este tipo de prácticas. Veamos cada uno de estos aspectos separadamente.

\subsection{La identificación de las competencias es el resultado de un "acuerdo" sobre el trabajo}

La identificación de las competencias no es un reflejo inmediato del trabajo efectivamente realizado por los JU, sino más bien, un acuerdo sobre determinados aspectos que la empresa considera relevantes. El proceso de seleccionar qué competencias han de evaluarse y cuál es el alcance de los cambios que conllevan este tipo de prácticas depende de las relaciones de fuerza existentes entre los distintos actores sociales.

En el caso estudiado, el actor determinante en la definición y las condiciones de gestión de las competencias ha sido la dirección de recursos humanos, lo que muestra el poder del actor empresarial para introducir modificaciones parciales en la relación de empleo a través de las políticas gestión de recursos humanos. Si bien

\footnotetext{
${ }^{5} \mathrm{JU}$ de Montaje.
} 
los sindicatos no han intervenido en la definición y selección de las competencias clave, su papel ha sido importante tanto en los aspectos que han sido objeto de negociación colectiva, por ejemplo, la creación de la figura del Jefe de Unidad de Primera, como en la creación de un clima de opinión contrario a la "lógica de la competencia". En general, la valoración de los sindicatos ha sido mayoritariamente crítica en lo que se refiere a las consecuencias que comporta el uso de las competencias en las relaciones sociales de trabajo y en la pérdida de garantías y derechos colectivos que se derivan de su uso (véase también apartado 4.2). A pesar de ello, la respuesta colectiva de los sindicatos ha sido en general débil y escasamente articulada, marcada por la ausencia de movilizaciones. Ello se debe, por un lado, a la mayor relevancia que para los sindicatos supone la profesionalización del grupo obrero (en un duro proceso de negociación en el momento de realizar el estudio) que implicará nuevas reglas de movilidad vertical y determinación del salario base. Por otro lado, para las principales confederaciones (UGT, CCOO y CGT) el hecho de que el colectivo afectado sea el de los mandos intermedios, y que el referencial no esté regulado mediante convenio colectivo, hace que su introducción sea una cuestión secundaria, o al menos, en la que han tenido pocas posibilidades intervenir.

Como hemos mencionado, los sindicatos valoran negativamente el referencial de los JU, esto, es un registro de las actividades específicas que han de desarrollar los Jefes de Unidad, por someter la promoción y remuneración de los mandos intermedios al juicio su mando jerárquico directo, sin la intervención del sujeto colectivo y excluyendo todo tipo de criterios impersonales negociados colectivamente, aplicados a todos los miembros de una misma categoría laboral. Esta personalización de la relación salarial, implica una primacía del derecho individual frente al principio de defensa de los derechos colectivos (tradicionalmente más eficaz), en el que se asienta la acción de las distintas confederaciones sindicales. Sin duda, para éstas el aspecto más relevante de esta personalización es el posible sesgo arbitrario implícito en la evaluación de las competencias.

Así mismo, el sindicato corporativo de mandos intermedios considera positivo crear un "horizonte profesional" para los JU pero, sin embargo, reconoce la existencia de diversos conflictos que han surgido a raíz de la aplicación de la lógica de la competencia. Ello se debe por un lado, a que el JU y el JU de Primera tienen las mismas responsabilidades, aún cuando se sitúen en niveles jerárquicos diferentes; y por otro, al carácter generalista del listado de competencias, que no contempla la especificidad de cada taller.

Los posibles sesgos arbitrarios derivados de la ausencia de una regulación y, sobre todo, de una negociación colectiva del uso de las competencias, podría neutralizarse mediante la participación de los sindicatos y los trabajadores afectados en la elaboración del referencial e introduciendo un cambio radical en los métodos y técnicas empleados en el proceso de su identificación y evaluación, en la línea que se viene apuntando en este artículo. En este sentido cabe recordar que la unidad de análisis del estudio de las competencias es precisamente de naturaleza relacional, lo que conllevaría una evaluación simultánea del trabajador, el equipo y la organización en que desarrolla su trabajo. 


\subsection{La competencia se inscribe en la situación y el contexto en que se desa- rrolla: Las condiciones de desempeño y evaluación}

La definición de la noción de competencia que proponemos hace especial hincapié en el carácter situado y contextualizado de dicho concepto. Ello conlleva que la evaluación e identificación de las competencias no sea una tarea exenta de dificultad puesto que exige un conocimiento y una percepción directa no sólo del desempeño del trabajo rutinario, sino también de aquellos momentos en que se han de afrontar diversas situaciones de carácter profesional y extra profesional de la persona evaluada.

Por otro lado, en este artículo se ha planteado una visión de la competencia vinculada y por tanto como atributo del grupo o comunidad de trabajo. Ello no supone la negación de la competencia como atributo individual, sino la afirmación de que su identificación y evaluación ha de ser inseparable del contexto social en el que se construyen, representan y se ponen en práctica dichas competencias, y de la situación en que se desarrollan (Boreham, 2004) y de ello es inseparable el equipo de trabajo.

El referencial elaborado por la empresa está basado en un registro exhaustivo de las actividades que han de desarrollar los JU. La filosofía de dicho referencial es coherente con la idea de que las competencias se identifican en la acción y situación de trabajo, pero contrariamente a tal desiderata, y como se ha expuesto anteriormente, el referencial de competencias se aproxima más a un listado de actividades que no de competencias en un sentido estricto.

A favor del modelo propuesto por la empresa, se ha de señalar que permite potencialmente una evaluación más equitativa al existir numerosos indicadores que permiten validar la realización de una determinada actividad. Sin embargo, dicho referencial no refleja satisfactoriamente la especificidad de cada taller dado que éste prioriza el objetivo de unificar y estandarizar los niveles de profesionalización, antes que el reconocimiento de las competencias específicas de los JU.

Así, el papel que ocupaba en Montaje todo lo relacionado con la gestión de los recursos humanos, lo ocupa en Soldadura la gestión de la calidad (relacionada con el producto final) y el control de la fiabilidad (que afecta a las máquinas, su mantenimiento, limpieza, etc) que constituyen un verdadero "caballo de batalla".

A su vez, las competencias identificadas por la empresa y los métodos de evaluación utilizados, no dan cuenta de la complejidad de situaciones y lógicas de acción desarrolladas en el ejercicio del proceso de trabajo, en el que intervienen no sólo las competencias del JU en cuestión, sino también las reglas que emergen de la red de actividades que se desarrollan en la factoría. De esta manera, la actividad del JU depende de la cooperación formal e informal y del correcto funcionamiento de las llamadas funciones soporte (mantenimiento, logística, control de calidad, ingeniería de métodos y departamento de recursos humanos), así como también de las UET anteriores y posteriores al proceso productivo desarrollado en la unidad analizada.

A su vez, son numerosas las dificultades que los JU encuentran a la hora de cumplir con determinadas exigencias recogidas en el listado de competencias. Si 
bien están plenamente conformes en general, con las prácticas de profesionalización, consideran que las principales dificultades para alcanzar los objetivos propuestos residen en la escasez de recursos y medios disponibles para desarrollar todo lo relativo a la gestión de la mano de obra (como por ejemplo el plan de formación de los operarios o el ajuste cualitativo de la plantilla). Al mismo tiempo, dicha ausencia de medios se refiere también a la falta de soporte de las funciones transversales, como mantenimiento, calidad o logística, con las que el JU ha de trabajar diariamente de manera estrecha y coordinada. Una carencia que también se manifiesta en la falta de apoyo de los superiores jerárquicos a la hora de resolver los problemas cotidianos de trabajo.

Esta postura se debe en gran parte a su posición estructural como mandos intermedios. De hecho, para asumir la intensidad de los ritmos de trabajo y los niveles de responsabilidad a los que está sometido este colectivo, la interiorización y apropiación subjetiva de los objetivos de productividad de la empresa, parece postularse como un mecanismo necesario. Como recuerda Burawoy (1979), la antigüedad en una empresa no sólo favorece la adquisición de conocimientos técnicos, sino también la interiorización de la cultura empresarial hecha de comportamientos no escritos y vínculos simbólicos.

\section{Conclusiones}

El caso analizado en este artículo muestra el alcance de las transformaciones que conlleva utilizar las competencias como evaluación y clasificación de los trabajadores, herramienta de promoción profesional y criterio de diferenciación salarial. Siguiendo a Pierre Rolle (2004), podemos afirmar que la competencia puede interpretarse como una expresión incipiente de una transformación en profundidad de las normas de empleo y las formas productivas. Dicha transformación supone la introducción de reglas personalizadas en la regulación de la relación salarial, una descentralización de los sistemas de control del trabajo, acorde con el creciente protagonismo del management, y un desarrollo sin precedentes de fórmulas que buscan la implicación de los trabajadores.

Tal y como se ha mostrado en este artículo, la selección de las competencias constituye un proceso complejo de interpretación caracterizado por la traducción del registro de la actividad al registro de las capacidades requeridas para desarrollar dicha actividad. En el caso estudiado el referencial de competencias ha constituido fundamentalmente, un registro de las actividades específicas que han de desarrollar los Jefes de Unidad, en cualquiera que sea el taller e incluso factoría considerada. Sin embargo, los conceptos de trabajo y actividad no comparten la misma naturaleza que el concepto de competencia. Esta última se manifiesta a través de la acción realizada, pero no constituye la acción en sí misma, sino el conjunto y combinación de los conocimientos, aptitudes y actitudes puestos en práctica para desarrollar tal acción y de una determinada manera. En este punto, se hace evidente la dificultad inherente a la hora de diseñar un método que establezca de manera adecuada el 
vínculo entre las competencias, las actividades realizadas y los indicadores que permitan evaluar a las primeras. Un vínculo que, en el caso estudiado, no se ha conseguido de manera satisfactoria.

A su vez se han analizado las consecuencias no deseadas derivadas de omitir la denominada "dimensión social de la competencia", referida a la importancia de hacer partícipes a los distintos actores afectados directa o indirectamente, así como a la necesidad de evaluar simultáneamente el individuo y la organización en la que desarrolla su trabajo. En este sentido es posible afirmar, siguiendo a Alaluf (2003), que evaluar las competencias no implica necesariamente una nueva modalidad de reconocimiento del trabajo, sino el control y evaluación de aquellas competencias que la empresa considera útiles en un contexto dado.

\section{Bibliografía}

Alaluf M. (2003). La sociología del trabajo a través de una relectura de Claude Durand. Cuadernos de Relaciones Laborales. Vol. 21 Num. 2, 93- 102.

Alonso L.E. (2007). La crisis de la ciudadanía laboral. Barcelona: Antropos.

Barney J. (1991). Firm resources and sustained competitive advantage. Journal of Management. Vol. 17 Num 1, 99- 120.

Bellier S. (2002). Le savoir-étre dans l'entreprise. Paris: Vuibert.

Bernard A. et al. (1998). La compétence éclatée dans les effets d'organisation. Le dileme américain: developer ou recruter les competences? Ressources Humaines. Une gestion éclatée (: 135-158). Paris: Económica.

Blanco Prieto A. (2007). Trabajadores competentes. Introducción y reflexiones sobre la gestión de recursos humanos por competencias. Madrid: ESIC Editorial.

Boam R., Sparrow P.R. (Eds.) (1992). Designing and achieving competency: a competencybased approach to developing people and organizations. Londres: McGraw- Hill.

Boltanski L., y Chiapello E. (2002). El nuevo espiritu del capitalismo. Madrid: Akal.

Brunet I. (2007). Calidad y recursos humanos del sector hotelero en Cataluña. Revista Internacional de sociología, 47, 73- 98.

Burawoy M. (1979). Manufacturing consent. Changes in the labour process under monopoly capitalism. Londres: The University Chicago Press.

Castillo J. J (1998). A la búsqueda del trabajo perdido. Madrid: Tecnos. 
—/et al. (2000). División del trabajo, cualificación, competencias. Una guía para el análisis de las necesidades de formación para los trabajadores. Sociología del trabajo. Nueva época 40, 3-50.

CEOE (2002). La gestión por competencias en España. Informe para el observatorio europeo de gestión por competencias. Departamento de Relaciones Laborales, CEOE.

Combes M. C. (2004). Identifier la dimension collective des compétences pour gérer le travail. Seminario ANACT. París, 13-14 de octubre.

Coller X. (2005). Estudio de casos. Madrid: Centro de investigaciones sociológicas. Fernández Rodríguez, C.J (2007). El discurso del management: Tiempo y narración. Madrid: CIS.

Fletcher, S. (1997). Analysing competence: Tools and techniques for analysing jobs, roles and functions. London: Kogan Page.

García J. Et al (2005). Una introducción al trabajo como relación social. En Lo que el trabajo esconde. Materiales para un replanteamiento sobre el trabajo (pp 19105). Madrid, Traficantes de sueños.

Giorgini P. (2000). La compétence au coeur du compromis social. En Bellier S. (Dir.), Compétences en action (pp 43- 71). Paris: Editions Liaisons.

Grant R. M. (1995) Dirección Estratégica. Conceptos, Técnicas y Aplicaciones. Madrid: Civitas.

Gómez Rodríguez C; López Aranguren E. (2004). La retórica del cambio en las organizaciones. Madrid: CIS

Grugulis I; Warhurst C; Keep E. (2004). What's happening to skill? En Grugulis I; Warhurst C; Keep E. (Ed). The skills that matter (pp 1-19). London: Palgrave Mcmillan.

Hamel G.; Prahalad C.K. (1994). Competing for the future. Boston: Harvard Business School Press.

Hammer M; Champy J. (1993). Reengineering the corporation: a manifesto for a business revolution. Londres: Nicholas Brealey Publishing.

Homs O; Prieto C. (1991). La construcción social de los requerimientos cualificacionales en las empresas españolas. Revista de Economía y Sociología del Trabajo, 13-14, 218- 224.

Jiménez A., (Coord.) (2000). Creando valor a través de las personas. Una guía para la gestión de las personas en el umbral de la e- sociedad. Madrid: Ediciones Díaz de Santos. 
Kanter R. (1999). Las nuevas fronteras del Management. Barcelona: Paidós. Lawler, E. (1994). From job-based to competency-based organizations. Journal of organizational behavior, 3- 15 .

Levy-Leboyer, C. (2002). Gestión de las competencias. Cómo analizarlas. Cómo evaluarlas. Cómo desarrollarlas. Barcelona: Ediciones Gestión 2000.

Lichtenberger I. (2003). Compétence, compétences. En Allouché, J. (Coord.), Encyclopédie des ressources humaines. Paris: Editions Vuibert.

Lozares C. et al. (1998). El tratamiento multiestratégico en la investigación sociológica. Papers, 55, 27- 43.

- (2007). Las unidades de análisis, sus contextos y sus modos de interacción en los procesos de trabajo: un estudio de caso. Papers 83, 83- 122.

McClelland D.C. (1973). Testing for competence rather than for intelligence. American Psychologist. Vol. 28, Núm. 1, 1-14.

Massó M. (2007). La utilización de las competencias como herramienta de gestión de los Recursos Humanos: el caso de las auxiliares de enfermería de un servicio hospitalario. Sociología del Trabajo 59. Nueva época: 79- 102

- (2009). La qualificació a debat: Una anaàlisi dels usos empresarials de la noció de competencia a Espanya. Barcelona: Consell de Treball Econòmic i Social de Catalunya.

Miguélez F. (1993). Clasificación, movilidad y relaciones laborales. Revista de economía y sociología del trabajo 21-22, 8- 18.

Nonaka I. (2000). La empresa creadora de conocimiento. En AA.VV, Gestión del conocimiento. Bilbao: Ediciones Deusto.

Oiry E. \& D'Iribarne A. (2001). La notion de competence: continités et changements par rapport à la notion de qualification. Sociologie du Travail, 43, 49-66.

Oiry E. (2003). De la qualification à la compétence. Rupture ou continuité? Paris: L'Harmattan.

Olivier N. et al. (2001). Trends in production and product development in the Japanese automotive industry. International journal of automotive technology and management Vol.1, 53-60.

Peters T. (1993). Reinventando la excelencia, el Management liberador. Barcelona: Ediciones B.

Ragin CH. \& Becker H. (1992). What is a case? Exploring the foundations of social inquiri. Nueva York: Cambridge University Press. 
Reynaud J-J. (2001). Le management par les competences: un essai d'analyse. Sociologie du Travail, 43, 7-31.

Rolle P. (2004). Asir y utilizar la actividad humana. Cualidad del trabajo, cualificación y competencia. En VVAA Lo que el trabajo esconde. Materiales para un replanteamiento del análisis sobre el trabajo (pp 197- 208). Madrid: Traficantes de Sueños.

Stake R. (1994). Case study. En Denzin, N., Lincoln Y. (Ed.). Handbook of qualitative research. London: Sage.

Stoof A et al. (2002). The boundary approach of competence: a constructivist aid for understanding and using the concept of competente. Human resource development review. Vol 1, Num 3, 345-365.

Stroobants M. (2005). La mutación al servicio del sistema productivo", en VVAA Lo que el trabajo esconde. Materiales para un replanteamiento del análisis sobre el trabajo (pp 173-192). Madrid: Traficantes de Sueños.

Tanguy L (Ed), (1986). L'introuvable relation formation/ emploi. Un état des recherches en France. Paris: La Documentation Française.

Tremblay M., Chênevert D. (1998). ¿Y si se remunerasen las competencias y no los puestos de trabajo? AEDIPE 5, 31- 38.

Valles M. S. (1997). Técnicas cualitativas de investigación social. Reflexión metodológica y práctica profesional. Madrid: Síntesis.

Van Loo J., Semeijn J. (2004). Defining and mesuring competences: an application to graduate surveys. Quality \& Quantity, 39, 331- 349

Willmont H. (2007). La fuerza es la ignorancia, la esclavitud es la libertad: la gestión de la cultura en las organizaciones modernas. En Fernández Rodríguez C., (Ed.), Vigilar y organizar (PP 103- 160). Madrid: Siglo XXI.

Womack J. P. et al. (1996). La máquina que cambió el mundo. Madrid : MacGraw- Hill.

Yin R. K. (1994). Case study research. Design and methods. Londres: Sage. Zarifian P. (1999). Objectif competence. Pour une nouvelle logique. Paris: Editions Liaisons. 